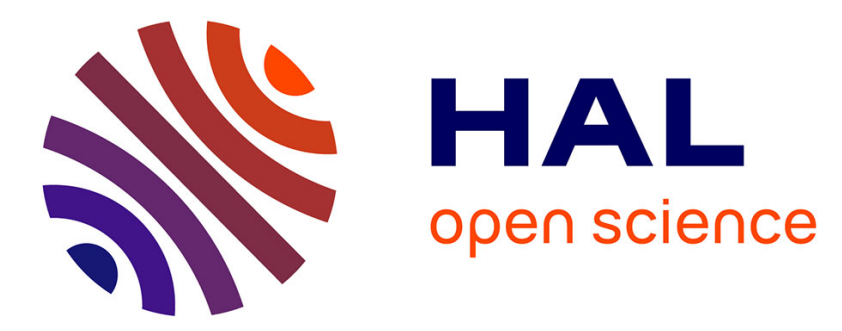

\title{
Modeling of Exposure to Low Frequency Electromagnetic Fields of Workers in Arbitrary Posture
}

Alice Conchin Gubernati, Fabio Freschi, Luca Giaccone, Riccardo Scorretti, Laurent Seppecher, Grégory Vial

\section{- To cite this version:}

Alice Conchin Gubernati, Fabio Freschi, Luca Giaccone, Riccardo Scorretti, Laurent Seppecher, et al. Modeling of Exposure to Low Frequency Electromagnetic Fields of Workers in Arbitrary Posture. IEEE Transactions on Magnetics, 2019, 56 (2), 10.1109/TMAG.2019.2949391 • hal-02415266

\author{
HAL Id: hal-02415266 \\ https://hal.science/hal-02415266
}

Submitted on 17 Dec 2019

HAL is a multi-disciplinary open access archive for the deposit and dissemination of scientific research documents, whether they are published or not. The documents may come from teaching and research institutions in France or abroad, or from public or private research centers.
L'archive ouverte pluridisciplinaire HAL, est destinée au dépôt et à la diffusion de documents scientifiques de niveau recherche, publiés ou non, émanant des établissements d'enseignement et de recherche français ou étrangers, des laboratoires publics ou privés. 


\title{
Modeling of Exposure to Low Frequency Electromagnetic Fields of Workers in Arbitrary Posture
}

\author{
Alice Conchin Gubernati ${ }^{1}$, Fabio Freschi ${ }^{1}$, Luca Giaccone ${ }^{1}$, \\ Riccardo Scorretti ${ }^{2}$, Laurent Seppecher ${ }^{3}$, Grégory Vial ${ }^{3}$ \\ ${ }^{1}$ Dipartimento Energia "G. Ferraris", Politecnico di Torino, Italy \\ ${ }^{2}$ Lab. Ampère, CNRS UMR 5005, École Centrale de Lyon, 69134 Ecully, France \\ ${ }^{3}$ Université de Lyon, CNRS UMR 5208, École Centrale de Lyon, ICJ, Ecully, France
}

\begin{abstract}
At present time, numerical dosimetry has reached a certain level of maturity and dedicated commercial software are already available. However, fast and accurate characterization of exposure in real condition is still challenging, among other reasons, because the exact posture of the "victim" has to be taken into account. The classical approach is to evaluate the source magnetic field and then to perform a dosimetric computation with a postured phantom. We propose a different approach, based on a change of variable, which takes a not postured phantom to a postured one. We demonstrate that for some rigid transformation this procedure does not end up in a change of tissue conductivity, which is localized in knees, elbows and other articulations, where deformations due to posturing are large. Only the source term will need to be determined by modifying the original one through a suitable transformation.
\end{abstract}

Index Terms-Computational phantoms, numerical dosimetry, low frequency magnetic fields, posture.

\section{INTRODUCTION}

$\mathbf{E}$ XPOSURE of workers to non ionizing (low frequency) electromagnetic fields is a source of concern and it is addressed by EU Directives 2013/35/UE [1]. In some cases, mitigation to reduce human exposure to electromagnetic fields is not sufficient (spot welding systems [2], [3]) or even impossible (e.g. MRI systems). In these cases dosimetric computations are mandatory to assess the respect of limits. These computations are usually slow, and require the knowledge of the model of the electromagnetic field source and the posture of the exposed worker.

In these years several realistic and detailed anatomical whole-body models of different types of human beings (e.g., male, female, children, pregnant woman, fat man,...) have been developed [4]. However, most of them were only available in the standing position with their arms along their sides. This greatly limited the possibility to study the electromagnetic safety in realistic exposure situations. For this reason, postured phantom based on models with the upright configuration started to be developed such as the sitting one [5] or the one with outstretched arms [6]. Posture transformation are based on maintaining internal tissues and organs continuity. Some methods to posture existing phantom have been developed, such as in [7] where a procedure for a posture transformation of anatomically realistic whole-body models has been proposed and a software to construct an arbitrary posture model has been created. These postured phantoms allowed to carry out the evaluation of human exposure to electromagnetic fields in realistic scenarios. However, posturing computational phantoms is a cumbersome task [7], [8], which necessarily introduces some approximations.

Manuscript received December 1, 2012; revised August 26, 2015. Corresponding author: A. Conchin Gubernati (email: alice.conchin@polito.it
The purpose of this paper is to demonstrate that from a numerical point of view the evaluation of human exposure to low frequency electromagnetic fields does not require a postured phantom. We propose a completely different approach based on a change of the magnetic field direction, that allows to perform all the dosimetric computations on a nonpostured numerical phantom, maintaining the same level of approximation of the postured model.

\section{Methods}

Geometrical transformations are well known in computational electromagnetics [9], [10]. Generally speaking, a change of variable leads to a fictitious modification of material properties, which depends on the Jacobian matrix of the transformation. In this section the differences between the classical approach and the proposed one are highlighted. In particular, we focus on the role played by the Jacobian matrix in the considered transformations.

\section{A. Classical approach}

As underlined in Section I, in the classical approach the phantom assumes the required posture to try to reproduce a real scenario. Since in low frequency (LF) numerical dosimetry the induced currents into the human body are too weak to modify the source field, human exposure to electromagnetic field problems can be solved by using the finite integration technique (FIT) using the electric scalar potential as unknown. This method can be seen as the extension of the scalar potential finite difference to tetrahedral meshes [11]. In the algebraic framework, the FIT is given by:

$$
\mathbf{G}^{\mathrm{T}} \mathbf{M}_{\sigma} \mathbf{G} \varphi=-\mathbf{j} \omega \mathbf{G}^{\mathrm{T}} \mathbf{M}_{\sigma} \mathbf{a}
$$

where $\mathbf{G}$ is the edge-to-node incidence matrix, $\mathbf{M}_{\sigma}$ is the conductance matrix, $\varphi$ is the electric scalar potential, and $\mathbf{a}$ is 
the integral of the magnetic vector potential due to the sources along the mesh edges [12].

In most cases, numerical dosimetry is made starting from data or measurements, and therefore from the knowledge of the magnetic flux density. Details about solving this type of problem can be found in [13].

\section{B. Proposed Method}

Since in the proposed method the phantom is not postured, formulation (1) must be rewritten by considering a new tissue conductivity and a new source term. Assume that the map $f: \Omega \mapsto \Omega_{p}$ takes the unpostured body $\Omega$ to the postured one $\Omega_{p}$. Hereafter, the subscript $p$ indicates quantities which depend explicitly on the posture of the body. Formulation (1) can be rewritten as:

$$
\mathbf{G}^{\mathrm{T}} \mathbf{M}_{\sigma_{\mathrm{p}}} \mathbf{G} \varphi=-j \omega \mathbf{G}^{\mathrm{T}} \mathbf{M}_{\sigma} \mathbf{a}_{\mathrm{p}} .
$$

This change of variable simplifies computations because now (2) refers directly to the unpostured body, eliminating the posturing step. On the other hand, elements dependent on the effect of posture, i.e. tissue conductivity tensor $\mathbf{M}_{\sigma_{\mathrm{p}}}$ and the source term $\mathbf{a}_{\mathrm{p}}$, must be determined [10]. Both these terms depend on the Jacobian matrix $\mathbf{J}_{p}$ of the map $f$ :

$$
\mathbf{M}_{\sigma_{\mathrm{p}}}^{\mathrm{loc}}=\mathbf{J}_{p}^{-1} \mathbf{M}_{\sigma}^{\mathrm{loc}} \mathbf{J}_{p}^{-\mathrm{T}} \cdot\left|\mathbf{J}_{p}\right| \quad ; \quad \mathbf{a}_{p}=\mathbf{J}_{p}^{\mathrm{T}} \mathbf{a} .
$$

It is important to underline that $\mathbf{M}_{\sigma_{\mathrm{p}}}^{\text {loc }}$ is locally computed and then the domain-based matrix $\mathbf{M}_{\sigma_{\mathrm{p}}}$ is assembled in a classical fem-like fashion. The Jacobian matrix is linked to the applied transformation.

\section{Human Body Transformations}

The mechanic movements that human body performs while it moves are manifold such as flexion, extension, rotation, abduction, adduction and circumduction. All these movements have to be reproduced using geometric transformations when the posture phantom is created. The geometric transformations that can be used are translation, rotation, and stretching.

Translation and rotation belong to the isometry group, i.e., they are distance-preserving transformations between metric spaces. In particular, the Jacobian matrix of translation is the identity matrix. This means that in (3) it does not make any contribution and, so, the tissue conductivity matrix can be rewritten as

$$
\mathbf{M}_{\sigma_{\mathrm{p}}}^{\mathrm{loc}}=\mathbf{M}_{\sigma}^{\mathrm{loc}}
$$

Instead, since rotation is a direct isometry, its Jacobian matrix is orthogonal, i.e. $\mathbf{J}_{p}^{\mathrm{T}}=\mathbf{J}_{p}^{-1}$, and $\left|\mathbf{J}_{p}\right|=1$. In most practical cases the local conductivity is isotropic, so $\mathbf{M}_{\sigma}^{\text {loc }}=\sigma \mathbf{I}$. For this reason, the tissue conductivity tensor described in (3) can be rewritten as

$$
\begin{aligned}
\mathbf{M}_{\sigma_{\mathrm{p}}}^{\mathrm{loc}} & =\mathbf{J}_{p}^{-1} \mathbf{M}_{\sigma}^{\mathrm{loc}} \mathbf{J}_{p}^{-\mathrm{T}} \cdot\left|\mathbf{J}_{p}\right|=\mathbf{J}_{p}^{\mathrm{T}} \sigma \mathbf{I} \mathbf{J}_{p} \cdot\left|\mathbf{J}_{p}\right| \\
& =\mathbf{J}_{p}^{\mathrm{T}} \sigma \mathbf{I} \mathbf{J}_{p}=\sigma \mathbf{J}_{p}^{\mathrm{T}} \mathbf{J}_{p}=\sigma \mathbf{I}=\mathbf{M}_{\sigma}^{\mathrm{loc}}
\end{aligned}
$$

We can therefore conclude that in the rotation case the tissue conductivity does not change and only the source term undergoes a transformation.

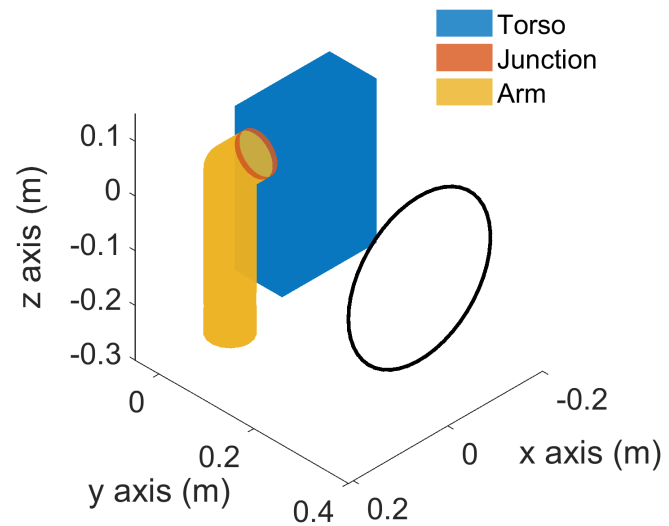

Fig. 1: Exposure scenario in three-dimensional domain considered in this paper. The three regions (torso, junction zone and arm) are highlighted by different colours.

Stretching is usually not considered in the posture phantom creation because much more importance is given to maintain the internal tissues and organs continuity and mass rather than to introduce physiological concepts, such as muscle contraction. Moreover, stretching produces so small deformations that its transformation matrix is approximately equal to the identity one. In this case the tissue conductivity matrix becomes

$$
\mathbf{M}_{\sigma_{\mathrm{p}}}^{\mathrm{loc}} \simeq \mathbf{M}_{\sigma}^{\mathrm{loc}}
$$

In general using (2)-(3) instead of (1) simplifies computations to a large extent. The key point is that in practice the transformation $f$ can be approximated as a piecewise rigid motion and applied to the source field instead of the computational domain. In fact, when posturing human body strong deformations are localized in articulations (elbows, knees, etc.): internal organs are only slightly deformed and brain is not deformed at all. For this reason, we have focused our attention on applying the proposed method to rotation transformation in the two-dimensional and three-dimensional case.

\section{TEST CASES}

The new approach described in Section II is tested on a two-dimensional and three-dimensional domain.

For the sake of simplicity, the two-dimensional domain $\Omega$ consists in an ellipse with center in the origin of the reference system. The minor semi-axis is on the $x$-axis with length $1 \mathrm{~m}$, while the major semi-axis is on the $y$-axis and it is long $1.2 \mathrm{~m}$. The tests are performed by considering an infinite vertical wire along $z$ as source field placed at a distance of $1.6 \mathrm{~m}$ from the center of the ellipse on the left side. The operating frequency is $50 \mathrm{~Hz}$ and the current flowing through the wire is $1 \mathrm{kA}$.

Instead, in the three-dimensional domain, to compare the in situ electric field obtained by using the classical approach and the new one, a simplified phantom exposed to a quasistatic magnetic field is considered. It is composed of three regions (Fig. 1): the torso, the arm and the junction zone, that connects the arm to the torso. The torso is a parallelepiped 


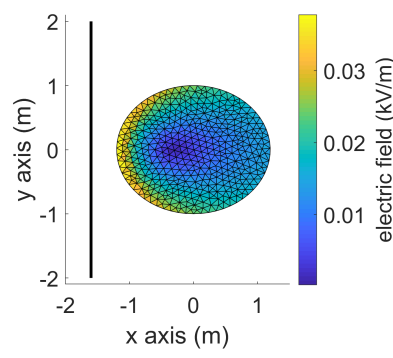

(a) electric field distribution on $\Omega_{p}$ (b) electric field distribution on $\Omega$
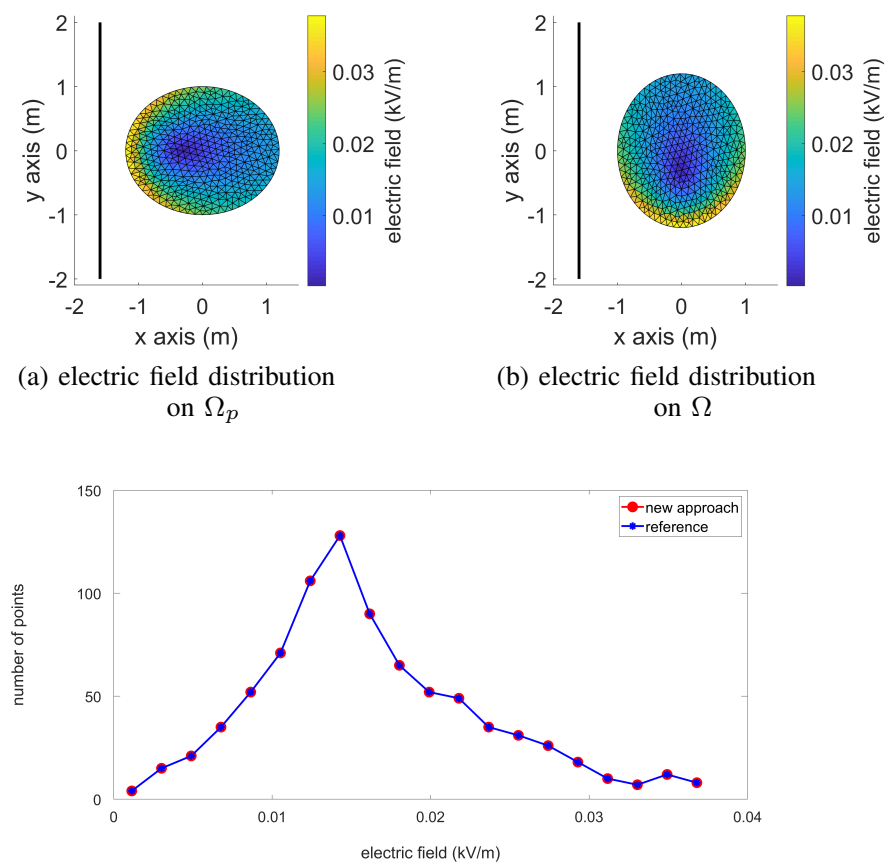

(c) Comparison of induced electric field between the reference case and the proposed method

Fig. 2: Reference solution for the induced electric field (a), solution obtained with the proposed method (b), electric field frequency diagram for both solutions (c).

with dimension $20 \times 10 \times 30 \mathrm{~cm}$, the junction is a cylinder with a $4 \mathrm{~cm}$ radius and $1 \mathrm{~cm}$ length, and the arm is formed by a cylinder equal to the junction connected to another cylinder with the same radius and $34 \mathrm{~cm}$ high. The tissue conductivity $\sigma$ is $0.2 \mathrm{~S} / \mathrm{m}$ for each tissue.

A one-loop coil with radius of $15 \mathrm{~cm}$ is located $35 \mathrm{~cm}$ from the torso and it is centered in the center of the shoulder. The axis of the coil is the y-axis. The operating frequency is $50 \mathrm{~Hz}$ and the current flowing through the coil is $1 \mathrm{kA}$.

The tetrahedral mesh in the simplified phantom consists of about 33000 nodes and 178000 tetrahedra (divided into about 67000 in the torso, 64000 in the junction zone, and 47000 in the arm). Moreover, the mesh resolution is $1 \mathrm{~mm}$ in the junction zone and $15 \mathrm{~mm}$ in the torso and arm.

\section{NumERICAL RESUlTS}

In this section, the numerical results obtained in the twodimensional and three-dimensional domain described in section III are reported and analysed. In the first case the ellipse is 90 degrees rotated, while in the second one the arm is 120 degrees rotated on the $y z$ plane.

\section{A. Two-dimensional domain}

The tests performed on the two-dimensional domain are based on a 90 degree rotation of the ellipse by considering as center of rotation the center of the ellipse.

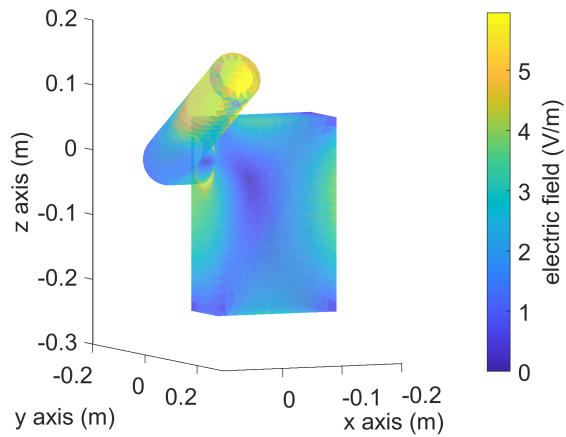

(a)

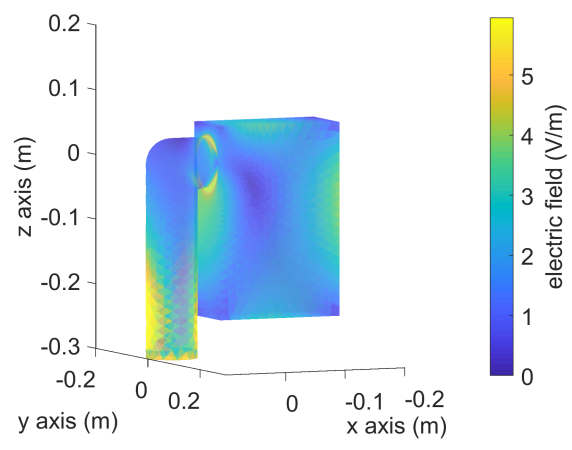

(b)

Fig. 3: Induced electric field distribution evaluated with the classical approach on the postured phantom (a) and with the proposed method on the non-postured phantom (b).

In the reference case (Fig. 2a), the ellipse is 90 degrees rotated and it is evident that the maximum exposure is on the left side, the closest one to the source. Fig. 2b, instead, shows the induced electric field distribution by using the proposed method. Since in the new approach the domain does not have to be transformed, the ellipse in not 90 degrees rotated. Although the vertical wire is kept in the same position, in Fig. 2b the ellipse maximum exposure is on the lower side. This highlights that the electric field direction generated by the wire has been changed thanks to equation (3) relative to the source term. Fig. 2c shows the electric field frequency diagram for both methods. It underlines that the results obtained with the proposed method (red curve) are exactly the same as those obtained with the classical approach (blue curve).

\section{B. Three-dimensional domain}

The classical approach (reference case) and the proposed method have been tested on the simple phantom presented in Section III, Fig. 1. As described in Subsection II-C, the geometric transformation used in the numerical simulations is determined by the rotation angle $\theta$ of the Jacobian matrix. In the three-dimensional domain it is: 1) equal to zero in the torso (no rotation); 2) equal to $\theta_{\max }=120^{\circ}$ in the arm; 3) linearly changes in the junction zone, where $\theta=\theta_{\max } \cdot \frac{x}{x_{\max }}$ with $x_{\max }=1 \mathrm{~cm}$. This linear variation is due to trying to recreate the arm real movement.

Since the one-loop coil is centered in the center of the shoulder, the phantom maximum exposure is at the end of the 


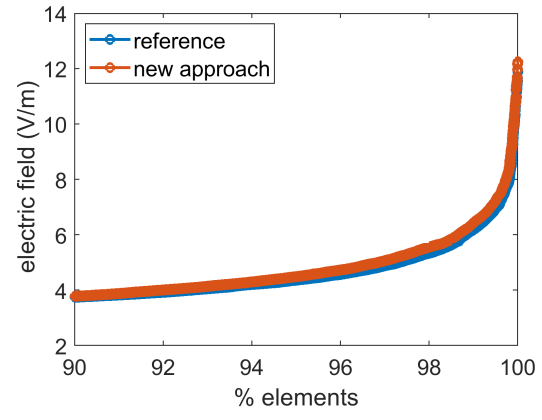

(a) Torso

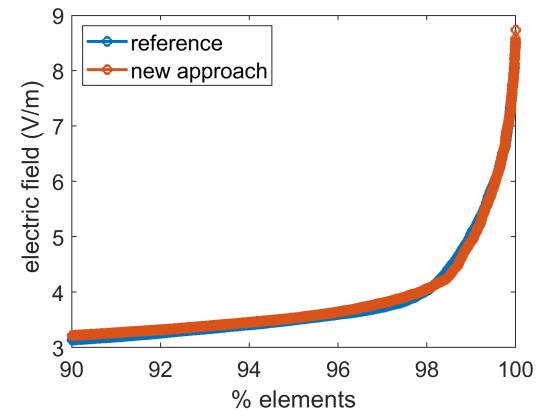

(b) Arm

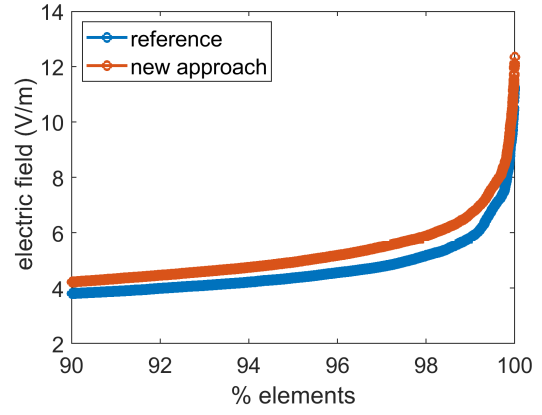

(c) Junction

Fig. 4: Comparison of the induced electric field between the two different methods in each components: torso (a), arm (b), and junction (c). Focus between the 90th and the 100th percentile value of the induced electric field.

rotated arm, as the reference case in Fig. 3a shows. Looking at Fig. 3b, we can say that the method works well because, if the classic approach were used, the maximum exposure should be in the centre of the shoulder in correspondence of the coil. Instead, the Fig. 3b shows that the highest induced electric field concentration is in the arm extremity, exactly as in the reference case (Fig. 3a). Fig. 4 also confirms these results. In fact, paying particular attention between the 90th and the 100 th percentile value of the induced electric field (maximum exposure), the results are completely comparable in the torso and in the arm, while there is a little deviation in the junction. The reason is that, while a complete rotation occurs in the arm, in the junction there is a piecewise rigid motion due to the linear variation of the Jacobian matrix angle. However, the largest deviation in the junction zone, evaluated as the ratio between the induced electric field value computed with the proposed method and the one computed with the classical approach, is $\sim 1.11$. It means that there is an overestimation of $11 \%$ (more than acceptable in numerical dosimetry).

\section{CONCLUSION}

A new approach to compute an approximation of induced electromagnetic field in the human body is proposed. The new method is based on the evaluation of human exposure to electromagnetic field by using a non-postured domain through a source term transformation. From a numerical point of view, this method guarantees simpler computations than the classical one because the use of phantom modified in the correct position, that can be different according to the situation, is not required.

Among all the geometric transformations, rotation best reproduces human body movements. Numerical results in two and three-dimensional domain related to this transformation have been reported in Section IV. In the two-dimensional domain, the results obtained with the proposed method are identical to the reference case. The reason is because rotation is an isometry (rigid transformation) and the use of a simple domain has allowed us not to introduce approximation errors. In the three-dimensional domain, the numerical results of the induced electric field distribution are comparable in the torso and arm, while in the junction there is an overestimation of the $11 \%$ between the 96 th and 100 th percentile due to the linear variation of the rotation angle. This overestimation is more than acceptable for a dosimetric assessment since these errors are comparable with the geometric ones due to the posture.

Our next work is to apply the proposed method to a more complicated phantom (e.g. Alvar [15]) in order to validate it.

\section{ACKNOWLEDGEMENT}

The authors would like to thank the PNREST Anses, $2018 / 1 / 242$, to have funded this project.

\section{REFERENCES}

[1] Directive 2013/35/UE of the European Parliament and of the Council, 26 June 2013.

[2] Canova, A., Freschi, F., and Giaccone, L. How Safe Are Spot Welding Guns to Use?: An Analysis of Occupational Exposure to Their Magnetic Field. IEEE Industry Applications Magazine, 24,3 (2018): 39-47.

[3] Giaccone, L., Cirimele, V., and Canova, A. Mitigation Solutions for the Magnetic Field Produced by MFDC Spot Welding Guns. IEEE Transactions on Electromagnetic Compatibility, in press.

[4] M.C. Gosselin et al., "Development of a new generation of highresolution anatomical models for medical device evaluation: the Virtual Population 3.0," Phys. Med. Biol., vol. 59, pp. 5287-5303, 2014.

[5] T.W. Dawson, "Numerical evaluation of $60 \mathrm{MHz}$ magnetic induction in the human body in complex occupational environments," Phys. Med. Biol., vol. 44, pp. 1025-40, 1999.

[6] T.W. Dawson, K. Caputa, and M.A. Stuchly, "Magnetic field exposures for UK live-line workers," Phys. Med. Biol., vol. 47, pp. 995-1012, 2002.

[7] Nagaoka, T. and Soichi W.. Postured voxel-based human models for electromagnetic dosimetry. Phys. Med. Biol. 53 (2008): 7047-7061.

[8] Gao, Jing. Generation of Postured Voxel-based Human Models Used for Electromagnetic Applications. Diss. Technische Universität, 2012.

[9] Nicolet, André, Zolla, F. and Agha, O. Geometrical transformations and equivalent materials in computational electromagnetism. Compel 27,4 (2008): 806-819.

[10] S.G. Johnson, Coordinate transformation and invariance in electromagnetism, $\quad$ https://math.mit.edu/ $\sim$ stevenj/18.369/spring08/ coordinate-transform.pdf.

[11] T. Dawson and M. Stuchly, "Analytic validation of a three-dimensional scalar-potential finite-difference code for low-frequency magnetic induction," Appl. Comput. Electrom., vol. 11, no. 3, pp. 72-81, 1996.

[12] F. Freschi, L. Giaccone, V. Cirimele, and A. Canova, "Numerical assessment of low-frequency dosimetry from sampled magnetic fields," Phys. Med. Biol., vol. 63, no. 1, p. 015029, 2018.

[13] A. Conchin Gubernati, F. Freschi, L. Giaccone, T. Campi, V. De Santis and I. Laakso, "Comparison of Numerical Techniques for the Evaluation of Human Exposure From Measurement Data," IEEE Trans. Mag., vol. 55, no. 6, 2019.

[14] Gaignaire, R., Scorretti, R., Sabariego, R.V., and Geuzaine, C. Stochastic uncertainty quantification of eddy currents in the human body by polynomial chaos decomposition. IEEE Trans. Mag. 48,2 (2012): 451-454.

[15] https://version.aalto.fi/gitlab/ilaakso/alvar 